\title{
Sustained Effectiveness of a Mandatory Employee Influenza Vaccination Policy at a Cancer Center
}

\author{
Martin C. Mahoney ${ }^{1}$, Janice Kozakiewicz ${ }^{2}$, Andrew D. Ray ${ }^{3}$ \\ ${ }^{1}$ Department of Medicine \& Employee Health Services, Roswell Park Cancer Institute, Buffalo, New York, USA \\ ${ }^{2}$ Employee Health Services, Roswell Park Cancer Institute, Buffalo, New York, USA \\ ${ }^{3}$ Department of Rehabilitation Science, School of Public Health \& Health Professions, Buffalo, New York, USA \\ Email: *Martin.mahoney@roswellpark.org
}

Received 8 August 2014; revised 8 September 2014; accepted 7 October 2014

Academic Editor: Praveen Kumar Amancha, Emory University, USA

Copyright (C) 2014 by authors and Scientific Research Publishing Inc.

This work is licensed under the Creative Commons Attribution International License (CC BY).

http://creativecommons.org/licenses/by/4.0/

(c) (i) Open Access

\section{Abstract}

Objectives: Despite the well-known advantages associated with immunization, it has proven difficult to achieve high levels of influenza vaccination among Health Care Professionals (HCPs). This report describes results of an influenza vaccination program implemented within a comprehensive cancer center. Methods: Using records from calendar years 2005-2013, we completed a retrospective analysis of annual influenza vaccination rates at this center. A mandatory influenza vaccination policy was implemented in 2010 , while prior to that vaccination was encouraged but not required. Vaccinations are free to employees and distributed at various locations. Annual influenza vaccination rates were examined by calendar year and by occupational group (medical, nursing, administrative, scientific, other support staff). Results: High levels of adherence with the mandatory influenza policy were observed for all employee groups. Prior to influenza vaccine mandates flu vaccination rates ranged from $\sim 30 \%-40 \%$ and increased to $85 \%-89 \%$ with the mandate. Conclusions: Robust influenza vaccination rates have been sustained since implementation of a vaccination mandate supporting further expansion of policies requiring influenza vaccination for HCPs.

\section{Keywords}

Influenza, Vaccination, Occupational Health, Health Care Workers, Mandatory Program, Health Policy

\footnotetext{
${ }^{*}$ Corresponding author.
} 


\section{Introduction}

Vaccination continues to be the primary approach to reduce the burden associated with seasonal influenza. It is estimated that seasonal influenza affects $\sim 5 \%-20 \%$ of the U.S. population and it is associated with as many as 250,000 hospital visits and up to 36,000 deaths annually [1]-[4]. Vulnerable populations, including the elderly and persons with comorbidities, are at increased risk for infection and upon becoming ill, they are at increased risk of complications and death. The total direct and indirect costs attributable to seasonal influenza is formidable and has been estimated as $\$ 87.1$ billion (range, $\$ 47$ billion - $\$ 150$ billion in US dollars) [5].

Numerous government and professional agencies have recommended that health care facilities should adopt an influenza vaccination program as a primary prevention strategy against the spread of influenza between health care personnel (HCP) and patients. Despite the well-known advantages associated with immunization, it remains difficult to achieve high levels of influenza vaccination among HCP. National surveys suggest that influenza vaccine rates for HCP remain relatively low. National data demonstrate that influenza vaccination among HCPs has increased from 60\% - 64\% between 2009-2011 to 72\% for 2012-2013; however this is still substantially below the $90 \%$ target established by the CDC [6].

In response to the low national influenza vaccination rates among HCPs, health care facilities have begun to implement programs designed to improve influenza vaccination rates among employees, especially those with direct patient contact. Efforts used by health care facilities have included financial incentives, no cost vaccination, signing declination forms, educational messaging acknowledging the benefits and risks of vaccination, improving access to free vaccine during work hours, mobile vaccination carts, E-mail reminders, and calling personnel. However, these strategies have been associated with only marginal improvements in compliance among HCPs. The continual suboptimal completion rates for influenza vaccination among HCPs nationally emphasizes the need for new and improved approaches [6].

Vaccination of health care providers reduces nosocomial spread of influenza to patients [7] and can also reduce illness episodes and lost workdays among staff, as well as the spread of influenza to family members. CDC recommends that each hospital facility use its level of flu vaccination coverage as one measure in a patient safety/quality improvement program [8]. Moreover, the Joint Commission also endorses strategies to enhance immunization rates among HCPs.

In addition to potential reductions in illness episodes among vaccinated employees, the importance of mandatory influenza vaccination has also been argued from a patient safety perspective given the substantial annual public health burden of influenza disease, opportunity for transmission from HCP to patients, effectiveness of influenza vaccine and moral obligation(s) to protect patients [9]. Universal influenza vaccination for all persons $>6$ months of age has been recommended since 2010 [10].

The purpose of this retrospective analysis is to describe a successful influenza vaccination program which resulted in a compliance rate that averaged $\sim 30 \%$ - 35\% above the national average over multiple influenza seasons.

\section{Subjects and Methods}

Design: We performed a retrospective analysis using employee influenza vaccination records over multiple years (2005-2013) at a comprehensive cancer center in the northeast United States. Because the 2004-2005 influenza season was unusual due to an influenza vaccine shortage, we selected the 2005-2006 season as the beginning time period for this study.

Subjects: This study was completed at a comprehensive cancer center which employs $\sim 3200$ persons focused on both clinical care and research. This facility includes a 133 bed hospital with 5400 admissions and 205,000 ambulatory visits annually.

In September 2010, based upon the recommendation of a multidisciplinary working group, the Center Chief Executive Officer (CEO) endorsed an institutional policy mandating that faculty/staff involved in direct patient care had the option of either completing annual seasonal influenza vaccination or wearing an isolation mask upon entering a patient care area during the period of November 1 thru March 30 of each influenza season; this same policy has been reaffirmed annually with minor modifications. Our institutional IRB deemed this project to be a Quality Improvement/Quality Assurance activity and not human subject research for which approval was required.

Each season, the influenza vaccination policy has been communicated to all faculty and staff via educational messaging as part of a promotional campaign titled "Patients 1st" which emphasizes patient safety (e.g., protec- 
tion of uniquely vulnerable patient populations) and the importance of receiving the influenza vaccination. A letter from the CEO to all employees was prominently featured on the main internal web page, along with appeals at various employee forums and via email messages. Vaccinations are free to employees and are available in multiple locations at various times and dates.

Dependent variable: Completion of influenza vaccine was defined as the main outcome variable. Influenza vaccination was offered on site, without charge, at the occupational health office and at various locations in clinical and non-clinical areas across the campus. Employees did have the opportunity of providing documentation of vaccination at another location (e.g., by their personal physician) but this was rarely utilized $(<0.5 \%)$. Employees could opt to complete an annual declination statement based upon either medical or personal objections which then required wearing a paper surgical mask during all patient interactions throughout the entire influenza season.

Independent variables: Completion of influenza vaccination was examined by calendar year and by occupational group (medical, nursing, administrative, scientific, other support staff.)

Analyses: SPSS (Version 21.0, IBM SPSS Statistics) was used to facilitate descriptive analyses. Adherence with the influenza vaccine policy was defined as either completion of vaccination or completion of a signed declination form. Adherence rates (and 95\% confidence intervals [11]), were assessed for the 2010-2011 thru 20132014 influenza seasons. Annual influenza vaccination coverage rates were also calculated by year overall and by occupational group.

\section{Results}

Overall adherence with the influenza vaccination policy of completing either vaccination or a declination form over 4 influenza seasons, '10 - '11 through '13 - '14, was 96.3\% (95\% confidence interval 95.3\% - 98.1\%). High levels of adherence were observed for all employee groups (see Table 1); rates remained stable within all groups over this 4 year interval (data not shown).

As shown in Figure 1, the overall rates of flu vaccination among employees during the influenza seasons of '05 - '06, '06 - '07, '07 - '08, and '08 - '09 were 31.1\%, 35.7\%, 34.2\%, and 38.5\%, respectively. The '09 - '10 season was atypical due to both the H1N1 pandemic during August/September 2009 and the "temporary" NYSDOH mandate in place from July to October of that year, with influenza vaccination coverage that season at $76.4 \%$. Following implementation of an institutional vaccine requirement, the overall rate of influenza vaccination for the ' 10 - ' 11 season was $85.4 \%$ which represents an absolute increase of $9 \%$ over the observed rate during ' 09 - ' 10 . Coverage rates for the ' 11 - '12, '12 - '13, and '13 - '14 seasons have ranged between 85.3\% and $88.7 \%$. Moreover, inclusion of employees who completed a declination form increased rates of adherence with the influenza vaccination policy to between $94 \%$ and $99 \%$ for the ' 10 - ' 11 through ' 13 - '14 seasons.

Figure 2 presents influenza vaccination completion rates by employee group during the four influenza seasons since the vaccination mandate. The highest level of adherence was achieved by medical and nursing staff, as well as support personnel; vaccination completion rates have remained robust among all employee groups.

Table 1. Adherence to the employee influenza vaccine policy ${ }^{*}$, overall and by employee group, for 2010-2011 thru $2013-$ 2014 seasons.

\begin{tabular}{cccc}
\hline Employee group & $\begin{array}{c}\text { \# of flu shots completed or } \\
\text { declinations signed }\end{array}$ & \# of employees & Adherence Rate (95\% CI) \\
\hline Administrative & 866 & 895 & $96.8 \%(95.4-97.7)$ \\
Medical & 176 & 193 & $91.2 \%(86.4-94.4)$ \\
Nursing & 554 & 571 & $97.0 \%(95.3-98.1)$ \\
Scientific & 564 & 596 & $94.6 \%(92.5-96.2)$ \\
Support Services & 960 & 984 & $97.6 \%(96.4-98.4)$ \\
Overall & 3120 & 3239 & $96.3 \%(95.6-96.9)$ \\
\hline
\end{tabular}

\footnotetext{
*Adherence includes either completion of seasonal influenza vaccine or submission of a signed declination form.
} 


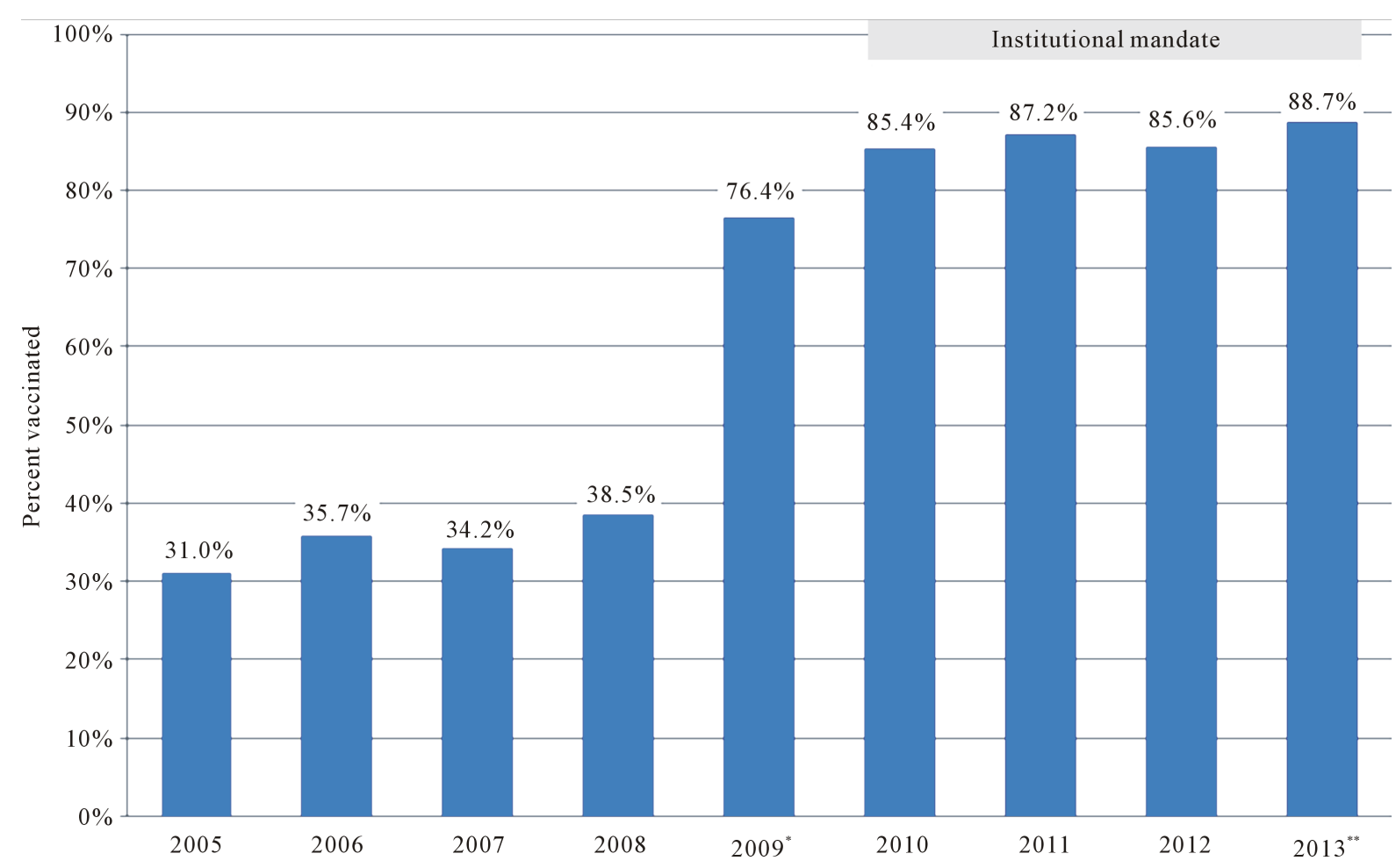

Figure 1. Overall influenza vaccination rates by year, 2005-2012. * In August 2009 H1N1 vaccination of health care providers (HCPs) was mandated by the New York State (NYS) hospital review and planning council, but suspended in October 2009 by the NYS Department of Health (NYSDOH). An institutional mandatory vaccination program was implemented in 2010. NYSDOH mandated vaccination of all HCPs in July 2013. The year below each bar refers to the influenza season that began that fall and extended to the following spring, e.g., "2005” represents the 2005-2006 influenza season.

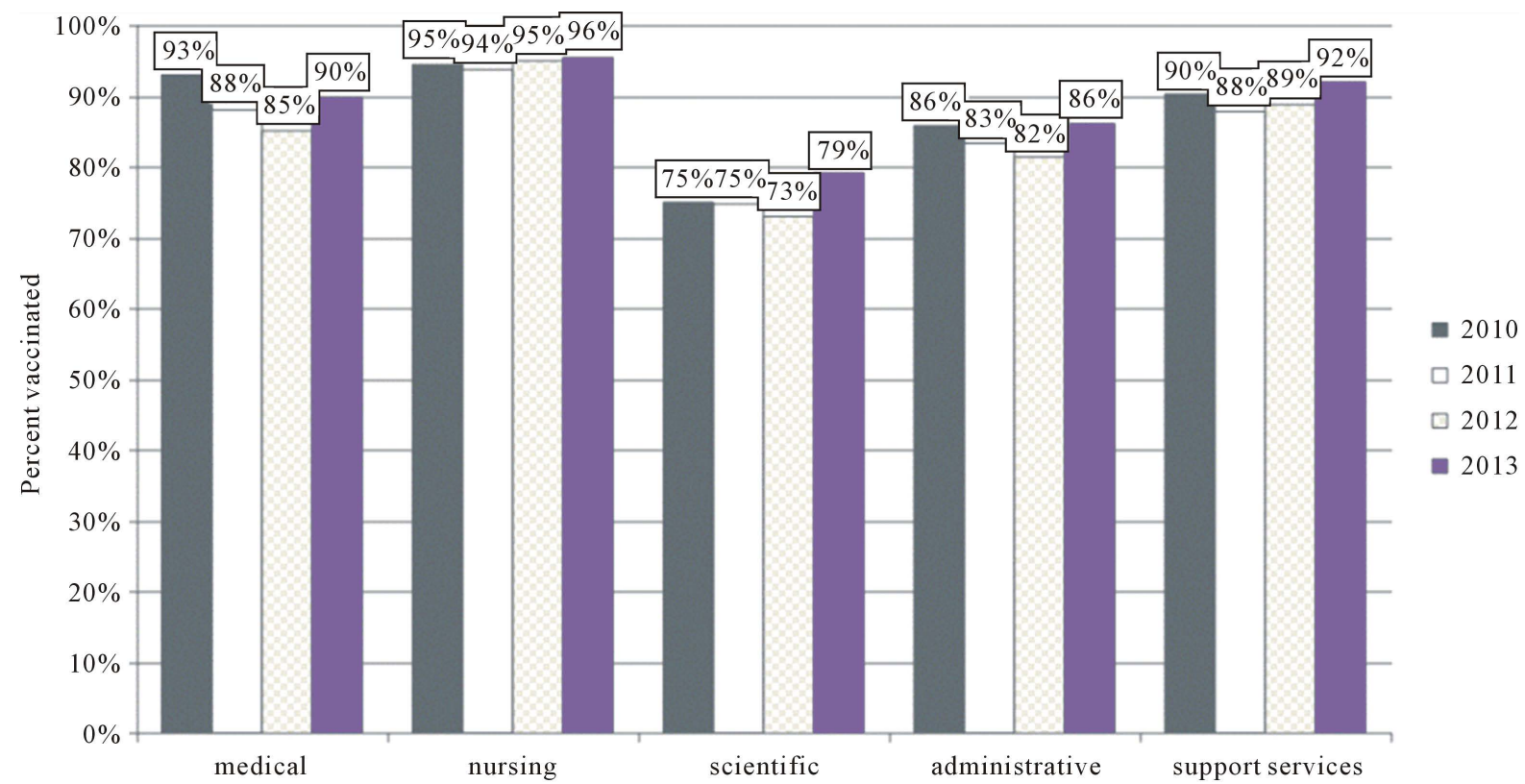

Figure 2. Influenza vaccination rates by year and employee group, 2010-2012. An institutional mandatory vaccination program was implemented in 2010. NYSDOH mandated vaccination of all HCPs in July 2013. The year refers to the influenza season that began that fall and extended to the following spring, e.g., "2010" represents the 2010-2011 influenza season. 


\section{Discussion}

This report describes results of an influenza vaccination program implemented within a comprehensive cancer center which achieved high levels of vaccination across several influenza seasons through use of an administrative mandate requiring vaccination for all employees with direct patient care responsibilities. Another noteworthy observation from this study is that the high levels of compliance with vaccination have been maintained consistently across several influenza seasons. It is hope that readers will be inspired to adapt these strategies in their own health care setting so that similar successes might be accomplished.

On occasion, mandates by state health boards and health care administrators, while offered with good intent, have been quickly reversed. In August 2009 the State Hospital Review and Planning Council (SHRPC), in New York State, mandated that health care facility personnel be vaccinated against both seasonal and H1N1 influenza. This emergency regulation was subsequently withdrawn on 11/11/09 due to concerns about a supply/distribution misalignment with both seasonal and H1N1 vaccines, as well as pending legal challenges from the New York State Nurses Association. In July 2013 the NYS Department of Health mandated influenza vaccine for all health care personnel.

(http://www.health.ny.gov/regulations/recently_adopted/docs/2013-07-31_prevention_of_influenza_transmissio n by healthcare_and_residential_facility and_agency_personnel.pdf)

Adherence with the influenza vaccination policy over 4 influenza seasons remained exceptional at $>96 \%$. Although subject to a ceiling effect, the influenza vaccination rates at our institution during the '10 - '11, '11 - '12, and '12 - ' 13 seasons, which ranged between $85 \%$ and $87 \%$, compared favorably with national coverage rates among HCPs of $64 \%, 67 \%$ and $72 \%$, respectively; in general, coverage rates were substantially higher among hospitals compared with other healthcare settings and among institutions having a vaccination requirement [6]. During '09 - '10 our institution achieved an overall influenza vaccination coverage rate of $76 \%$ among all employees compared to a vaccination rate of $39 \%$ during the prior season ('08 - '09). It is possible that some of this increase may have resulted enhanced awareness of influenza resulting from the 2009 H1N1 pandemic and/or the temporary department of health mandate which was rescinded in October 2010.

Preventing the transmission of infectious agents such as influenza in health care facilities is a difficult task and requires a multifaceted approach. Historically most health care institutions have relied upon educational messages emphasizing the benefits of influenza vaccination, issuing a general recommendation to their workers and providing vaccine at no cost on site. More recently, signed declinations, monitoring and feedback, the inclusion of influenza vaccination as a quality measure and vaccine mandates have been added [7] [8]. We believe that in additional to the mandated policy, our success has resulted from several factors: the provision of free vaccines delivered during multiple days/times, including at work locations; internal website promotional announcements promoting this as a patient safety initiative; and using supervisors to encourage vaccination among organizational units.

Individual health beliefs have been reported to be an important moderator for vaccination behaviors. Compared to healthcare personnel who do not complete influenza vaccination, those who are adherent with influenza vaccination generally view the vaccine as safe and effective, considered themselves and persons around them as more susceptible to influenza, considered influenza illness as significant, and felt that completing vaccination would help to protect others around them [12].

The higher than average adherence rates observed in this study may be associated in part with the high risk patients typically seen at this comprehensive cancer facility and the fact they are vulnerable to infection. A "patient first" mentality seems to be more apparent at facilities where HCP are working with high-risk patients [13]. Also, staff at facilities with higher than average HCP influenza vaccination rates (>78\%) typically cite patient protection as the second most important reason for vaccination, behind self-protection [14].

The Healthy People 2020 goal for influenza vaccination of healthcare personnel is $90 \%$

(http://healthypeople.gov/2020/topicsobjectives2020/objectiveslist.aspx?topicid=23) and efforts continue toward reaching that goal. Support is growing for mandatory influenza vaccination of health care workers [15] [16]. In December 2013, the Infectious Disease Society of America (IDSA), the Society for Healthcare Epidemiology of America (SHEA) and the Pediatric Infectious Disease Society (PIDS) issued a joint statement supporting mandatory immunizations consistent with Advisory Committee on Immunization Practices for health care professionals, including annual influenza vaccination.

(http://www.idsociety.org/uploadedFiles/IDSA/Policy and_Advocacy/Current_Topics_and_Issues/Immunizatio 
ns_and_Vaccines/Health_Care_Worker_Immunization/Statements/IDSA_SHEA_PIDS\%20Policy\%20on\%20M andatory\%20Immunization\%20of\%20HCP.pdf) The justification of vaccination mandates is grounded in obligations to prioritize the interests of patients, to "do no harm", to protect especially vulnerable populations, and to provide professionalism and leadership in the area of prevention [17]. Since 2007 the Joint Commission on Accreditation of Healthcare Organizations (JCAHO) has required accredited institutions to provide on-site influenza vaccine, track vaccination rates and promote efforts to increase coverage rates.

A 2011 survey of a sample of 998 acute care hospitals in the United States reported that $56 \%$ of responding institutions reported having an institutional requirement for influenza vaccination [18]. Moreover, many of these requirements appeared to fall short of recommendations by IDSA/SHEA/PIDS and other organizations and failed to include the breadth of health care personnel with a high likelihood of patient contact, including physicians, nurses, nursing assistants, therapists (occupational, physical, speech), technicians, emergency personnel, dental personnel, pharmacists, lab personnel, autopsy personal, social workers, students, trainees, contract staff and volunteers, as well as staff not directly involved in patient care, such as clerical, dietary, housekeeping, and maintenance staff, or volunteers working in mainly non-clinical roles who may potentially transmit infection to or from a HCP [19].

The use of "disincentives" to motivate completion of influenza vaccination has yielded mixed results. Data from 84 hospitals with consequences for flu vaccination non-adherence (masking, education, reassignment, termination) showed a $20 \%$ improvement in influenza vaccination rates compared and 66 hospitals without consequences where rates increased by $11 \%$ [20]. However, a larger study which included over 420 institutions found that the impact of hospital influenza vaccination policies specifying termination for non-adherence was comparable to policies requiring masking or reassignment for non-adherence; both types of policies were associated with increases in HCP vaccination rates of $12 \%$ - 13\% [21]. Interestingly these authors also concluded that state vaccination mandates were not associated with changes in influenza coverage rates among employees [21].

This analysis has documented the successful implementation of mandatory influenza policy among employees at a comprehensive cancer center. A unique strength of the present study is a review of experiences over a period of 4 influenza seasons. HCP vaccinated against influenza can lower health care costs since they experience fewer upper respiratory infections, have fewer sick visits to medical offices, use fewer sick days and use fewer medications for acute seasonal illnesses [22]. We are unable to assess the impact of influenza vaccination among this retrospective employee cohort since sick time usage includes family sick time, medical screening and other absences not directly attributable to employee illness.

High rates of compliance and increased rates of influenza vaccination among HCPs have been sustained since implementation of a mandatory influenza policy. These results suggest that mandatory influenza policies for HCPs can be successfully implemented in the setting of a comprehensive cancer center and can be maintained over several consecutive influenza seasons. It is hoped that this experience will lead to further expansion of requirement for annual influenza vaccination for HCPs and the application of similar policies in other health care facilities.

\section{References}

[1] Grohskopf, L.A., Shay, D.K., Shimabukuro, T.T., Sokolow, L.Z., Keitel, W.A., et al. (2013) Prevention and Control of Seasonal Influenza with Vaccines. Recommendations of the Advisory Committee on Immunization Practices-United States, 2013-2014. MMWR Recommendations and Reports, 62 (RR07), 1-43.

[2] Thompson, W.W., Shay, D.K., Weintraub, E., et al. (2003) Mortality Associated with Influenza and Respiratory Syncytial Virus in the United States. JAMA, 289, 179-186. http://dx.doi.org/10.1001/jama.289.2.179

[3] Thompson, W.W., Shay, D.K., Weintraub, E., et al. (2004) Influenza-Associated Hospitalizations in the United States. JAMA, 292, 1333-1340. http://dx.doi.org/10.1001/jama.292.11.1333

[4] Zhou, H., Thompson, W.W., Viboud, C.G., et al. (2012) Hospitalizations Associated with Influenza and Respiratory Syncytial Virus in the United States, 1993-2008. Clinical Infectious Diseases, 54, 1427-1436. http://dx.doi.org/10.1093/cid/cis211

[5] Molinari, N.A., Ortega-Sanchez, I.R., Messonnier, M.L., et al. (2007) The Annual Impact of Seasonal Influenza in the US: Measuring Disease Burden and Costs. Vaccine, 25, 5086-5096. http://dx.doi.org/10.1016/j.vaccine.2007.03.046

[6] Ball, S.W., Donahue, S.M.A., Izrael, D., Walker, D.K., DiSogra, C., Martonik, R., et al. (2013) Influenza Vaccination Coverage among Health-Care Personnel-United States, 2012-13 Influenza Season. Morbidity and Mortality Weekly Report (MMWR), 62, 781-786. 
[7] Maltezou, H.C. (2008) Nosocomial Influenza: New Concepts and Practice. Current Opinion in Infectious Diseases, 21, 337-343. http://dx.doi.org/10.1097/QCO.0b013e3283013945

[8] Shefer, A., Atkinson, W., Friedman, C., et al. (2011) Immunization of Health-Care Personnel: Recommendations of the Advisory Committee on Immunization Practices (ACIP). MMWR Recommendations and Reports, 60, 1-45.

[9] Poland, G.A., Tosh, P. and Jacobson, R.M. (2005) Requiring Influenza Vaccination for Health Care Workers: Seven Truths We Must Accept. Vaccine, 23, 2251-2255. http://dx.doi.org/10.1016/j.vaccine.2005.01.043

[10] Fiore, A.E., Uyeki, T.M., Broder, K., et al. (2010) Prevention and Control of Influenza with Vaccines: Recommendations of the Advisory Committee on Immunization Practices (ACIP), 2010. MMWR Recommendations and Reports, 59, $1-62$.

[11] Newcombe, R.G. (1998) Two-Sided Confidence Intervals for the Single Proportion: Comparison of Seven Methods. Statistics in Medicine, 17, 857-872. http://dx.doi.org/10.1002/(SICI)1097-0258(19980430)17:8<857::AID-SIM777>3.0.CO;2-E

[12] Harris, K.M., Uscher-Pines, L., Black, C.L., Euler, G.L., Singleton, J.A., et al. (2011) Influenza Vaccination Coverage among Health-Care Personnel—United States, 2010-11 Influenza Season. MMWR Morbidity and Mortality Weekly Report, 60, 1073-1077.

[13] Takayanagi, I.J., Cardoso, M.R., Costa, S.F., Araya, M.E. and Machado, C.M. (2007) Attitudes of Health Care Workers to Influenza Vaccination: Why Are They Not Vaccinated? American Journal of Infection Control, 35, 56-61. http://dx.doi.org/10.1016/j.ajic.2006.06.002

[14] Hakim, H., Gaur, A.H. and McCullers, J.A. (2011) Motivating Factors for High Rates of Influenza Vaccination among Healthcare Workers. Vaccine, 29, 5963-5969. http://dx.doi.org/10.1016/j.vaccine.2011.06.041

[15] Poland, G.A. (2010) Mandating Influenza Vaccination for Health Care Workers: Putting Patients and Professional Ethics over Personal Preference. Vaccine, 28, 5757-5759. http://dx.doi.org/10.1016/j.vaccine.2010.07.057

[16] Caplan, A. (2011) Time to Mandate Influenza Vaccination in Health-Care Workers. The Lancet, 378, 310-311. http://dx.doi.org/10.1016/S0140-6736(11)61156-2

[17] Caplan, A. and Shah, N.R. (2013) Managing the Human Toll Caused by Seasonal Influenza: New York State’s Mandate to Vaccinate or Mask. JAMA, 310, 1797-1798. http://dx.doi.org/10.1001/jama.2013.280633

[18] Miller, B.L., Ahmed, F., Lindley, M.C. and Wortley, P.M. (2011) Institutional Requirements for Influenza Vaccination of Healthcare Personnel: Results from a Nationally Representative Survey of Acute Care Hospitals-United States, 2011. Clinical Infectious Diseases, 53, 1051-1059. http://dx.doi.org/10.1093/cid/cir633

[19] Pearson, M.L., Bridges, C.B. and Harper, S.A. (2006) Influenza Vaccination of Health-Care Personnel: Recommendations of the Healthcare Infection Control Practices Advisory Committee (HICPAC) and the Advisory Committee on Immunization Practices (ACIP). MMWR Recommendations and Reports, 55, 1-16.

[20] Nowalk, M.P., Lin, C.J., Raymund, M., Bialor, J. and Zimmerman, R.K. (2013) Impact of Hospital Policies on Health Care Workers’ Influenza Vaccination Rates. American Journal of Infection Control, 41, 697-701. http://dx.doi.org/10.1016/j.ajic.2012.11.011

[21] Zimmerman, R.K., Lin, C.J., Raymund, M., Bialor, J., Sweeney, P.M. and Nowalk, M.P. (2013) Hospital Policies, State Laws, and Healthcare Worker Influenza Vaccination Rates. Infection Control and Hospital Epidemiology, 34, 854-857. http://dx.doi.org/10.1086/671265

[22] Nichol, K.L., Lind, A., Margolis, K.L., Murdoch, M., McFadden, R., Hauge, M., Magnan, S. and Drake, M. (1995) The Effectiveness of Vaccination against Influenza in Healthy, Working Adults. New England Journal of Medicine, 333, 889-893. http://dx.doi.org/10.1056/NEJM199510053331401 
Scientific Research Publishing (SCIRP) is one of the largest Open Access journal publishers. It is currently publishing more than 200 open access, online, peer-reviewed journals covering a wide range of academic disciplines. SCIRP serves the worldwide academic communities and contributes to the progress and application of science with its publication.

Other selected journals from SCIRP are listed as below. Submit your manuscript to us via either submit@scirp.org or Online Submission Portal.
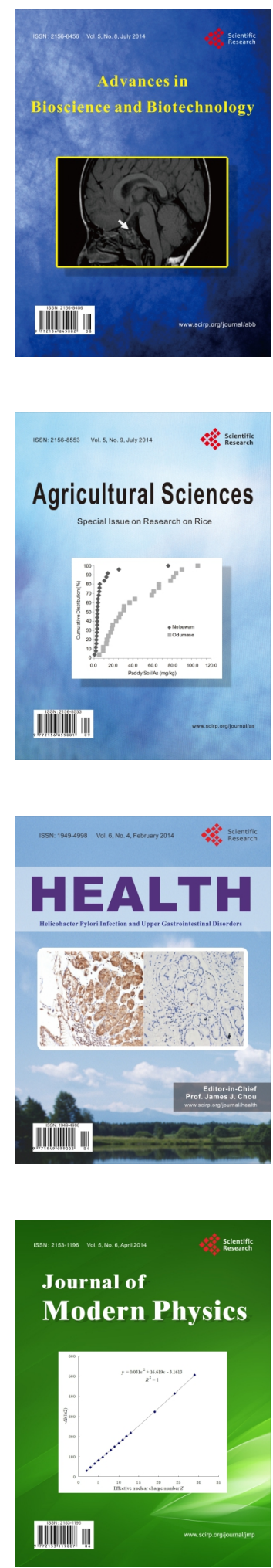
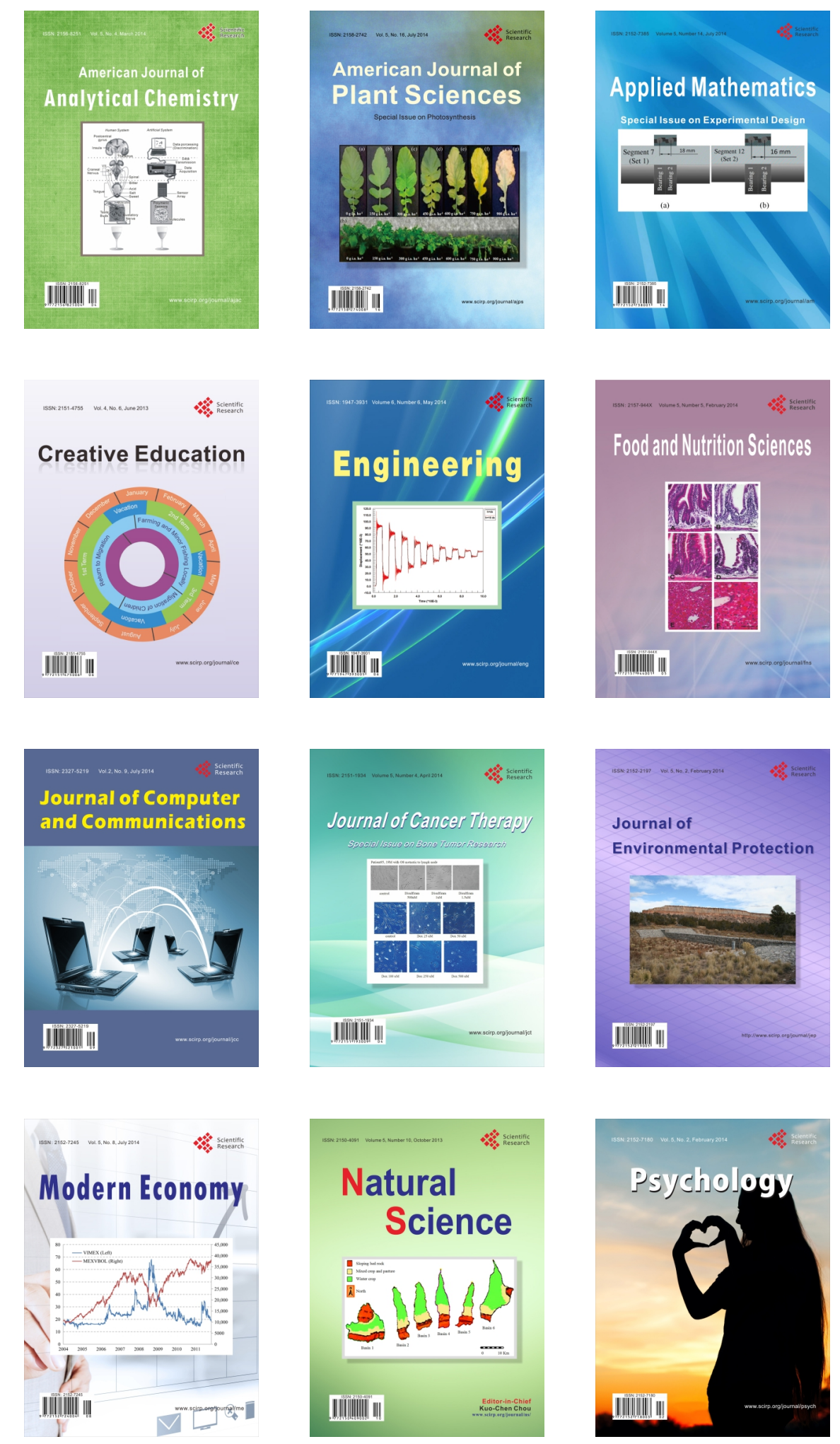